\title{
Untersuchungen zur automatisierbaren potenziometrischen Messung des Kaliumgehalts und des pH-Werts von Ackerproben vor-Ort
}

\author{
Manfred Decker ${ }^{1}$, Arnold Wezel ${ }^{2}$, Frank Gerlach $^{1}$, Petra Teichmann ${ }^{1}$, Martin Schneider ${ }^{3}$ und Winfried Vonau ${ }^{1}$ \\ ${ }^{1}$ Kurt-Schwabe-Institut für Mess- und Sensortechnik e.V. Meinsberg, Waldheim, Deutschland \\ ${ }^{2}$ Hochschule Mittweida, University of Applied Sciences, Mittweida, Deutschland \\ ${ }^{3}$ Agricon $\mathrm{GmbH}$, Ostrau, Deutschland \\ Kontakt: manfred.decker@ksi-meinsberg.de
}

\section{Einleitung}

Die ortsaufgelöste Analyse des Gehalts wichtiger Bodeninhaltsstoffe ist für den Landwirt eine wesentliche Voraussetzung zur Etablierung einer bedarfsgerechten Düngung seiner Ackerflächen. Insbesondere eine genaue Kenntnis des Kaliumgehalts und des $\mathrm{pH}$-Werts eröffnet inm die Möglichkeit, durch eine gezielte, an die Erfordernisse angepasste Versorgung des Bodens mit Düngemitteln, eine signifikante Steigerung des landwirtschaftlichen Ertrages zu realisieren. Die bisherige Bestimmung dieser Größen erfolgt nach der Probenahme und aufwändiger Probenvorbereitung im letzten Schritt mittels nasschemischer Analysen. Der arbeitsintensive Prozessablauf erlaubt keine zeitnahe Messwertermittlung vor-Ort. Darüber hinaus würde eine hohe räumliche Auflösung bei der Beprobung einen hohen finanziellen Aufwand erfordern. Aus diesen Gründen wird eine rasche, automatisierte Messung der Zielparameter noch vor-Ort auf dem Acker angestrebt.

\section{Problemstellung und Lösungsansatz}

Zur bedarfsgerechten Versorgung landwirtschaftlicher Nutzflächen mit Nährstoffen ist die Kenntnis des pH-Werts des Bodens sowie die Verfügbarkeit wichtiger Mineralien essentiell. Da diese Parameter schon innerhalb der Fläche eines Hektars deutlich voneinander abweichen können, wird eine hohe räumliche Beprobungsdichte angestrebt. Von der Firma Agricon $\mathrm{GmbH}$, Ostrau, wird eine automatisierte Lösung angeboten, mit der eine zuverlässige Bodenprobenahme auf abgeernteten Äckern möglich ist. Die Sammlung der Erdproben erfolgt maschinell mit einem Stecheisen, welches an einem geländegängigen Fahrzeug installiert ist und in definierten Abständen Bodenproben entnimmt. Die Einheit ist in der Abbildung $1 \mathrm{zu}$ sehen. Mit der vorgestellten Lösung wird zurzeit auf einer Fläche von 3 ha je eine Mischprobe gewonnen. Diese muss anschließend in einem gewerblichen Labor einer chemischen Analyse der Zielparameter unterworfen werden.
Im Rahmen der Entwicklung wird eine schnelle Analyse des pH-Werts und des Kaliumgehalts frisch gesammelter Ackerproben direkt auf dem Feld angestrebt. Zur parallelen Messung der Zielionen soll das potenziometrische Verfahren herangezogen werden. Die Verwendung dieser ionenselektiven Analyseme-

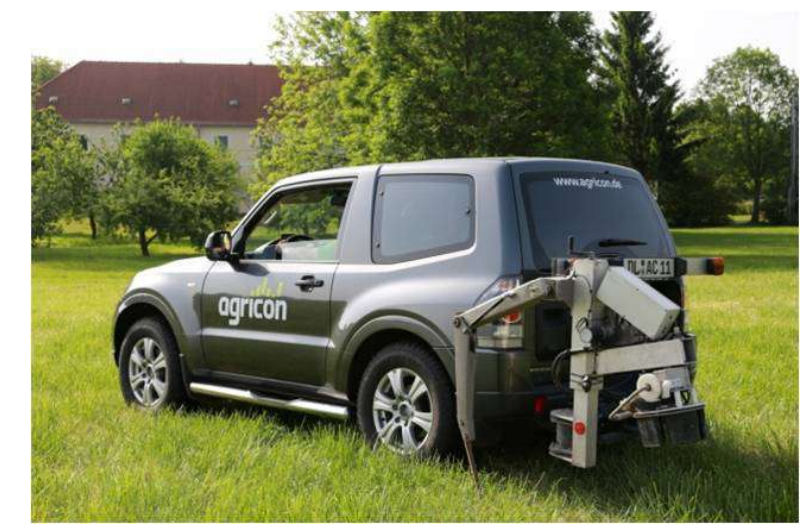

thode für beide Parameter gleichzeitig bietet den Vorteil, dass bei der Realisierung der Apparatur der technische Aufwand verringert werden kann.

Abb. 1: SUV der Firma Agricon GmbH mit Probensammeleinheit

Für die pH-Wert-Bestimmung in abrasiven Medien hat sich der Einsatz der potenziometrischen Antimonoxid-Elektrode bewährt [1]. Zentraler Baustein der Messzelle ist ein Antimonring, der in Kombination mit einer Referenzelektrode auf Basis des Systems $\mathrm{Ag} / \mathrm{AgCl}$ mit 0,01 molarer $\mathrm{CaCl}_{2}$-Lösung als Referenzelektrolyt, die $\mathrm{pH}$-abhängige Spannung zur Analyse des pH-Werts liefert. Die Kombination dieser potenziometrischen Bausteine erlaubt innerhalb von 30 Sekunden eine Bestimmung des pH-Werts. Bei der Durchführung der Analyse wird, angelehnt an das DIN-Verfahren [2], die Probe vor der Analyse gesiebt und mit einem definierten Volumen einer 0,01 molaren $\mathrm{CaCl}_{2}$-Lösung aufgeschlämmt. Dieser Messaufbau soll im Rahmen der Entwicklung um einen mechanisch stabilisierten Sensor auf Basis einer polymermatrixgestützten Kalium-selektiven Elektrode ergänzt werden, um eine simultane potenziometrische Bestimmung des Alkalimetalls zu ermöglichen. Zur mechanischen Stabilisierung der 
Membranen wird der Einsatz einer porösen Fritte oder die Integration eines Stahlnetzes untersucht. Die Eignung der Kalium-selektiven Elektroden soll an Realproben durch den Vergleich mit den Resultaten ermittelt nach dem standardisierten Verfahren der VDLUFA [3], aufgezeigt werden.

\section{Materialien und Methoden}

Kaliumchlorid, Reinheit $\geq 99,5 \%$, und Calciumchlorid-dihydrat $\geq 99 \%$, zur Herstellung der Kalibrierund Extraktionslösungen stammten von der Firma Roth $\mathrm{GmbH}$ und CoKG, Karlsruhe, Deutschland.

Die Herstellung der Kalium-selektiven Membranen erfolgte nach der Vorschrift von Kim et al. [4]. Kalium-lonophor I (Valinomycin), PVC (high molecular weight), Bis(2-ethylhexyl)sebacat, und Kaliumtetrakis(4-chlorophenyl)borat wurden von Sigma-Aldrich, Buchs, Schweiz, erworben, wie auch Tetrahydrofuran mit einer Reinheit $\geq 99,5 \%$ als Lösemittel für die Komponenten des ionenselektiven Cocktails. Zur Fertigung der Membranen wurde 2,2 $\mathrm{mL}$ des ionenselektiven Cocktails in einem Glasring mit einem Innendurchmesser von $24 \mathrm{~mm}$, platziert auf einer Glasplatte, ausgegossen und mit einem Filterpapier bedeckt. Nach der Verdunstung des Lösemittels innerhalb von zwei Tagen wurden die Membranen unter Verwendung des ionenselektiven Cocktails auf PVC-Röhrchen (Innendurchmesser 6,0 mm) aufgeklebt.

Zur mechanischen Stabilisierung der KaliumMembranen in porösen Glasstrukturen wurden VitraPor Spezialfilterplatten mit einer Porengröße von $160-250 \mu \mathrm{m}$ und einer Dicke von $2 \mathrm{~mm}$ der Firma ROBU Glasfilter-Geräte $\mathrm{GmbH}$, Hattert, Deutschland verwendet. Die Fritte wurde passend ausgeschnitten und in ein Glasrohr mit einem Innendurchmesser von $5 \mathrm{~mm}$ eingeschmolzen. Zur Füllung der Membran mit dem ionenselektiven Cocktail wurde dieser mit einer $5 \mathrm{~mL}$ Spritze langsam in die poröse Fritte eingegeben. Nach der Verdunstung des Lösemittels wurde noch mit einer Spritze weiterer Cocktail auf die poröse Struktur gegeben, um noch potenziell vorhandene Hohlräume zu schließen.

Alternativ wurden Kalium-sensitive Elektroden mit einem Edelstahlnetz stabilisiert. Dafür wurden Edelstahlsiebe mit einer Maschenweite mesh 400 der Firma Koenen $\mathrm{GmbH}$, Ottobrunn, Deutschland, mit einem Durchmesser von $6,0 \mathrm{~mm}$ ausgestanzt und im Glasring auf der Glasplatte platziert. Die weitere Herstellung der ionenselektiven Membran erfolgte nach dem schon beschriebenen Verfahren. Nach Verdunstung des Lösemittels umschloss die Membran vollständig das Edelstahlnetz. Die ausgestanzten Membranen konnten anschließend mit Hilfe des Cocktails fest mit dem PVC-Rohr verklebt werden.

Für die potenziometrischen Messungen wurde bei den Elektroden eine 0,01 molare Kaliumchlorid-
Lösung als Innenelektrolyt und ein elektrochemisch chloridisierter Silberdraht als Ableitelement verwendet. Die potenziometrischen Messungen erfolgten mit einem pH-Meter 764 Multi-Calimatic der Firma Knick Elektronische Messgeräte $\mathrm{GmbH}$ und CoKG, Berlin, Deutschland. Als Referenzelektrode diente ein Eigenbau des $\mathrm{KSI}$ mit AgAgCl-Ableitelement und einer 0,01 molaren $\mathrm{CaCl}_{2}$-Lösung als Innenelektrolyt. Die Elektrodeneigenschaften wurden nach dem Batchverfahren in gerührten Lösungen mit definierten Kaliumgehalten bei $25^{\circ} \mathrm{C}$ bestimmt.

Die Untersuchungen von Realproben erfolgten analog dem Verfahren der VDLUFA [3] zur ,Bestimmung von pflanzenverfügbarem Kalium und Natrium im Calciumchloridauszug auf Volumenbasis' [3]. Ein Teil der Proben wurde bei $37^{\circ} \mathrm{C} 24 \mathrm{~h}$ getrocknet und dann mit einem Sieb der Maschenweite $2 \mathrm{~mm}$ Grobpartikel abgetrennt. Abweichend zur Vorschrift wurde jedoch - in Anlehnung an die pH-Messung - nur eine 0,01 molare $\mathrm{CaCl}_{2}$-Lösung eingesetzt, während die Standardmethode eine 0,0125 molare $\mathrm{CaCl}_{2}$ Lösung zur Aufschlämmung vorgibt. Um den apparativen Aufwand bei der späteren Realisierung des Prototyps zur Messung beider Parameter zu reduzieren, sollte nur eine Extraktionslösung eingesetzt werden. Aus diesem Grund wurde auf die bei der pH-Bestimmung bewährte 0,01 molare $\mathrm{CaCl}_{2-}$ Lösung zurückgegriffen. Frisch gesammelte wie auch getrocknete Realproben wurden mit dem doppelten und dem zwölffachen Volumen des Extraktionsmittels versehen, eine Stunde gerührt und das Messpotenzial verfolgt. Zur Bestimmung der Konzentration diente das Potenzial nach 60 Minuten.

Nach Abschluss der Messung wurde die Lösung filtriert und eine Analyse flammenphotometrisch mit einem Atomabsorptionsspektrometer novAA 400 der Firma Analytik Jena AG, Jena, Deutschland, durchgeführt.

\section{Ergebnisse}

Die Kalium-selektiven Eigenschaften einer Membran auf Basis der gewählten Zusammensetzung kann der Abbildung 2 entnommen werden.

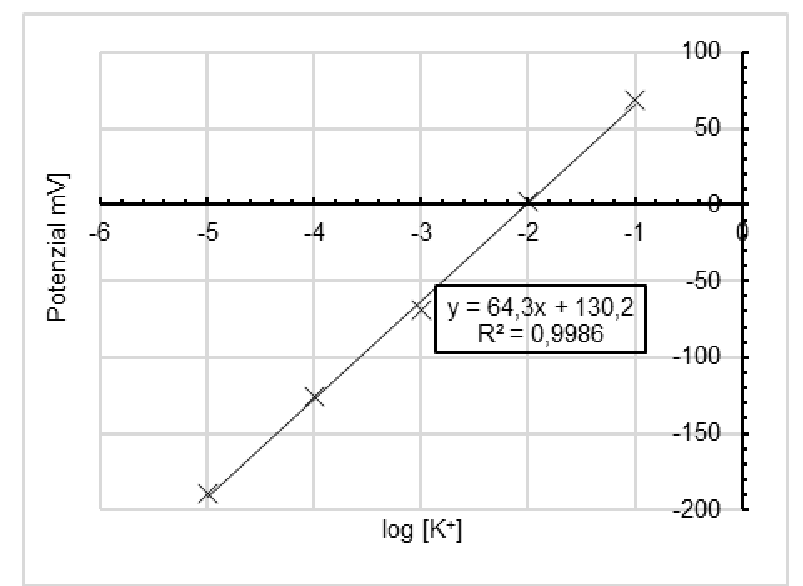


Abb. 2: Kalibrierung einer unstabilisierten Kalium-selektiven Membran (dest. Wasser, Batchverfahren, gegen $\mathrm{Ag} / \mathrm{AgCl}, 0,01 \mathrm{M} \mathrm{CaCl}_{2}$-Referenzelektrode)

Die Elektroden zeigen in Kombination mit der Referenzelektrode ein leichtes Supernernstverhalten von 64,3 mVIAD Kaliumionen im Aktivitätsbereich von $10^{-5}$ bis $10^{-1} \mathrm{~mol} / \mathrm{L} \mathrm{K}^{+}$, welches auf den Innenelektrolyten der Referenzelektrode zurückgeführt wird, der von einer 0,01 molaren Calciumchlorid-Lösung gebildet wird. Dennoch kann bei dem gewählten ionenselektiven Cocktail auf eine sehr gute Sensitivität für Kaliumionen geschlossen werden.

Analoge Untersuchungen wurden für die stabilisierten Elektroden durchgeführt. Abb. 3 zeigt eine mikroskopische Aufnahme einer polymermatrixgestützten Kalium-selektiven Elektrode, stabilisiert durch eine poröse Glasfritte.

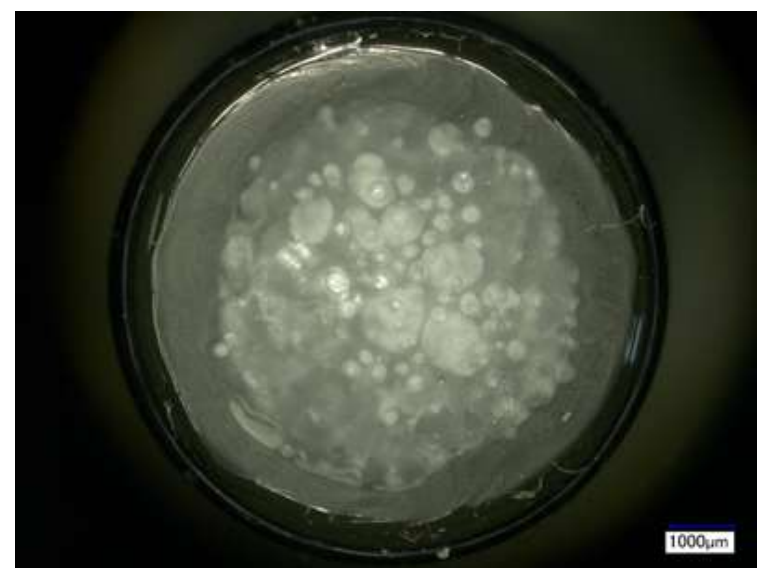

Abb. 3: Mikroskopische Aufnahme einer stabilisierten Elektrode mit Glasfritte und eingeschlossener Membran (30-fache Vergrößerung)

Die Elektrodeneigenschaften einer mittels Glasfritte verstärkten Kalium-sensitiven Elektrode können exemplarisch dem Graphen in Abbildung 4 entnommen werden. Mit dem Batchverfahren wird eine nur unbefriedigende Sensitivität auf Kaliumionen von 42,2 mVIAD erhalten.

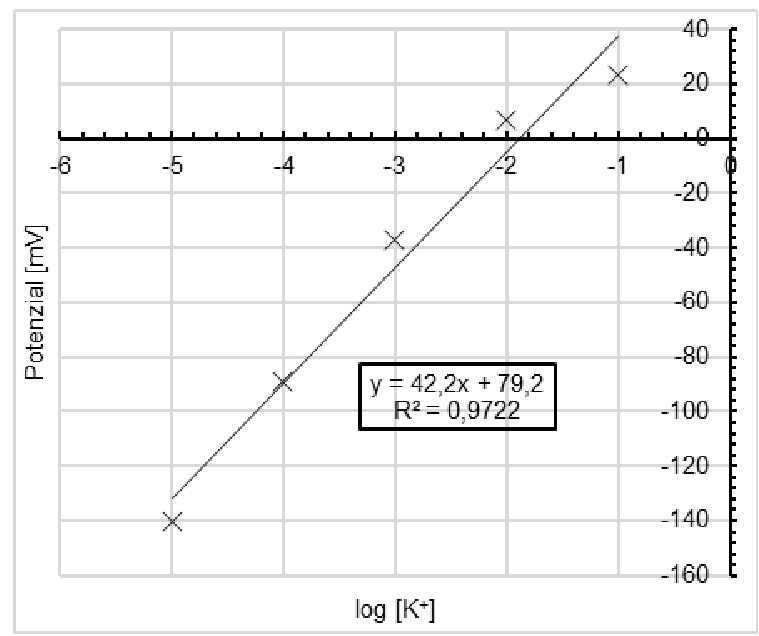

Abb. 4: Kalibrierung einer mittels Glasfritte stabilisierten Kalium-selektiven Membran (dest. Wasser Batchverfahren, gegen $\mathrm{Ag} / \mathrm{AgCl}, 0,01 \mathrm{M} \mathrm{CaCl}_{2}$-Referenzelektrode)

Abbildung 5 zeigt die Aufnahme einer Elektrode mit einer durch ein Edelmetallnetz stabilisierten Membran.

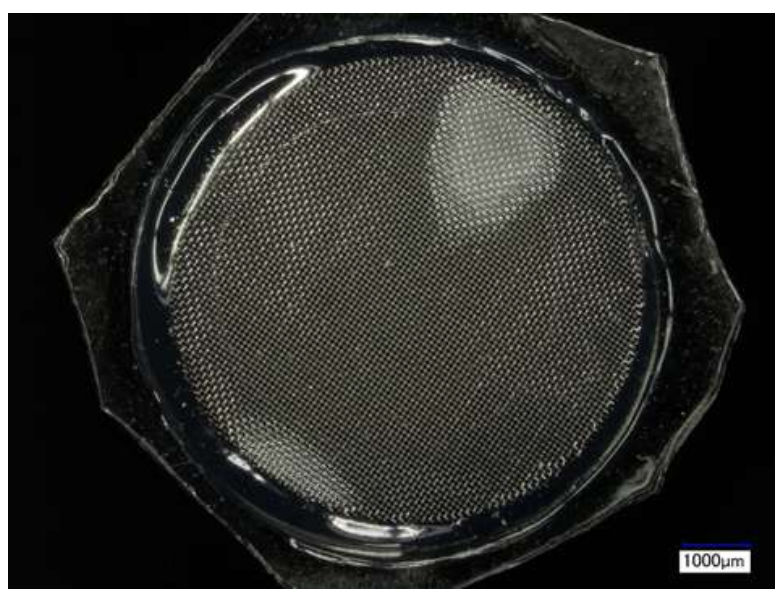

Abb. 5: Mikroskopische Aufnahme einer Kaliumselektiven Elektrode stabilisiert mit einem Edelstahlnetz (30-fache Vergrößerung)

Die mittels eines Edelstahlnetzes verstärkten Elektroden zeigten mit 63,8 mVIAD ein nahezu identisches Ansprechen auf die Kaliumionenaktivität im Vergleich mit den unstabilisierten Sensoren, wie dem Graphen in Abbildung 6 zu entnehmen ist.

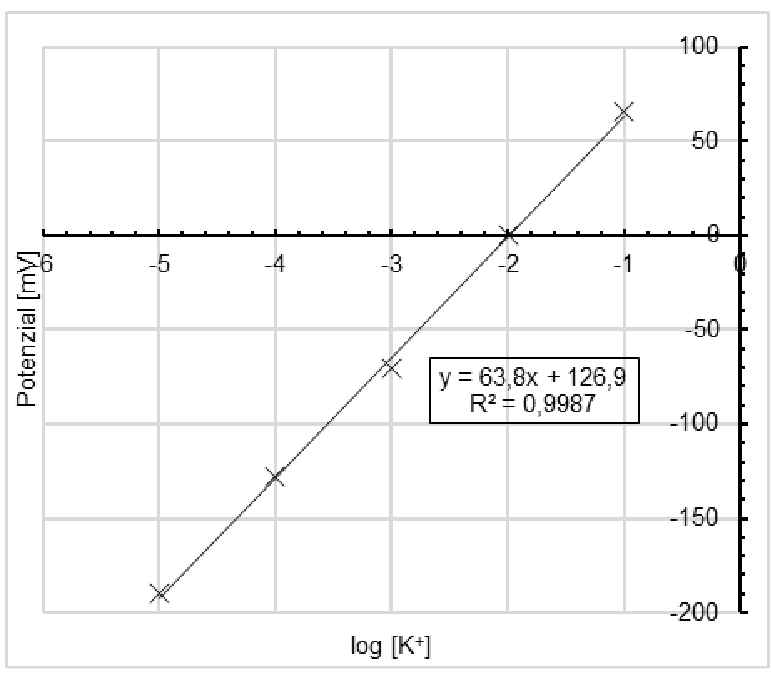

Abb. 6: Kalibrierung einer Edelstahlnetz-verstärkten Kalium-selektiven Membran (dest. Wasser; Batchverfahren, gegen $\mathrm{Ag} / \mathrm{AgCl}, 0,01 \mathrm{M} \mathrm{CaCl}_{2}$-Referenzelektrode)

Die Ergebnisse lassen den Schluss zu, dass eine mechanische Verstärkung der hochempfindlichen ionenselektiven Membranen durch die Integration eines Edelstahlnetzes möglich ist, ohne die Eigenschaften des Sensors zu beeinträchtigen. 
In ersten Messreihen wurde der Einsatz der ionenselektiven Kaliumelektroden bei der Bestimmung des verfügbaren Kaliums in Bodenproben untersucht. Dabei wurde der Kaliumgehalt potenziometrisch und mittels Atomabsorptionsspektrometer in Bodenproben ermittelt. Angelehnt an die Vorschrift der VDLUFA wurde zum einen das Volumen der Bodenprobe mit dem zwölffachen Volumen einer 0,01 molaren $\mathrm{CaCl}_{2}$-Lösung aufgeschlämmt. Da bei der pH-Messung mit der $\mathrm{Sb}_{2} \mathrm{O}_{3}$-Elektrode, um Extraktionsmittel zu sparen, schon das doppelte Volumen an Lösung für eine zuverlässige pH-Wertbestimmung ausreicht, wurde auch dieses Mischungsverhältnis in die Messreihen mit einbezogen. In Abbildung 7 sind exemplarisch die Messresultate für eine sandige Lehmprobe dargestellt. Dabei wurden sowohl Erdproben, die keiner Trocknung unterworfen wurden, als auch Böden, die zuvor $24 \mathrm{~h}$ bei $37^{\circ} \mathrm{C}$ getrocknet wurden, untersucht.

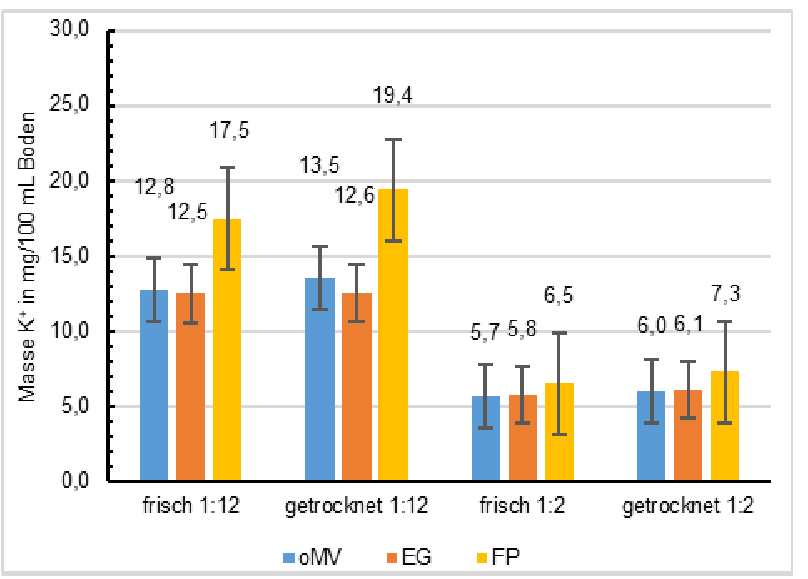

Abb. 1: $\mathrm{K}^{+}$-Gehalte einer Probe der Bodenart St3 (sandiger Lehm) potenziometrisch bestimmt mit einer unstabilisierten Membran (oMV), einer Edelstahlgitter-verstärkten Membran (EG) und mit Flammenphotometrie (FP) $(n=3)$

Die Messungen zeigten, dass im Vergleich mit den flammenphotometrischen Analysen die $\mathrm{K}^{+}$-selektiven Elektroden um 20 bis $30 \%$ niedrigere Kaliumgehalte ermitteln. Hingegen sind Abweichungen zwischen einem unstabilisierten Sensor und einer Membran, die mit einem Edelstahlgitter modifiziert wurde, nicht zu beobachten. Die Minderbefunde können damit erklärt werden, dass die ionenselektiven Sensoren nur die Aktivität der unkomplexierten $\mathrm{K}^{+}$-Ionen in der Lösung anzeigen, während die Flammenphotometrie die Gesamtheit aller Kaliumspezies erfasst.

Bei allen Analysemethoden ist der in der Bodenprobe ermittelte Kaliumgehalt stark abhängig vom Mischungsverhältnis Feststoff zu Extraktionsmittel. Bei der Zugabe von kleinen Volumina an $\mathrm{CaCl}_{2}-$ Lösung werden bezogen auf $100 \mathrm{~mL}$ Bodenprobe eine deutlich niedrigere Masse an frei verfügbarem Kalium beobachtet. Da die Größenordnung dieser Minderbefunde sowohl bei der Flammenphotometrie als auch bei der potenziometrischen Analyse ver- gleichbar sind, kann geschlossen werden, dass ein Großteil des Kaliums noch an den Bodenpartikeln immobilisiert ist und für die Messung in Lösung nicht zur Verfügung steht.

\section{Diskussion und Ausblick}

Den ersten Experimenten kann entnommen werden, dass sich die mechanische Stabilisierung einer ionenselektiven Membran mit einem Edelstahlnetz für den Einsatz in abrasiven Medien anbietet. Messungen in Lösungen der aufgeschlämmten Bodenproben zeigen keine Unterschiede zwischen unbehandelten und behandelten Sensoren. Im Vergleich zur Flammenphotometrie werden allerdings um 20 bis $30 \%$ geringere Kaliumgehalte ermittelt, was auf komplexgebundenes Kalium zurückgeführt wird.

Die bestimmten Kaliumgehalte in den Bodenproben sind bei der Verwendung kleiner Extraktionsvolumina deutlich geringer als bei den Vorgaben der VDLUFA. Dies wird darauf zurückgeführt, dass ein beträchtlicher Teil des Kaliums an den Bodenpartikeln gebunden vorliegt und erst bei der Zugabe weiterer $\mathrm{CaCl}_{2}$-Lösung in die Flüssigkeit übertritt.

In weiterführenden Versuchsreihen werden für zahlreiche Bodenproben und -arten Untersuchungen durchgeführt werden, um die Zusammenhänge zwischen den Bodenpartikeln, Mischungsverhältnissen und dem Vorliegen an freien Kalium aufzuklären. Potenziell kann ein Algorithmus zur Abschätzung des Kaliumgehalts ermittelt werden, der den gewünschten Einsatz geringer Volumina an Extraktionsmittel in der Praxis erlaubt. Weitere Versuchsinhalte werden darauf gerichtet werden, eine einfache Herstellung von stabilisierten $\mathrm{K}^{+}$-Sensoren für die angestrebte Aufgabenstellung zu realisieren.

\section{Literatur}

[1] DECKER, M.; BAUSE, S.; TEICHMANN, P.; SCHNEIDER, M.; VONAU, W.: Development of an automatic system for the on-site $\mathrm{pH}$-measurement of soil samples. In: tm - Technisches Messen 84 (2017), Nr. 12, S. $659-671$

[2] DIN ISO 10390: Bodenbeschaffenheit - Bestimmung des pH-Wertes. (DIN 10390:2005) DIN Deutsches Institut für Normung e.V. Berlin

[3] VDLUFA Methodenbuch Band 1: Die Untersuchung von Böden 6.2.1.6; Die Bestimmung von pflanzenverfügbarem Kalium und Natrium im Calciumchloridauszug auf Volumenbasis. (1997), VDLUFA Verlag, Darmstadt, S. 1 - 8

[4] KIM, H.J.; HUMMEL, J.W.; BIRRELL, S.J.: Evaluation of nitrate and potassium ion-selective membranes for soil macronutrient sensing. In: Transactions of the ASABE 49 (2006) Nr. 3, S. 597 - 606

\section{Danksagung}

Die vorgestellten Forschungsarbeiten sind Teil eines Projektes gefördert vom Europäischen Fond für 
Regionale Entwicklung (EFRE), FKZ 100351424.

Das Kurt-Schwabe-Institut für Mess- und Sensortechnik Meinsberg e.V. wird mitfinanziert durch Steuermittel auf der Grundlage des vom Sächsischen Landtag beschlossenen Haushaltes. 